\section{Contrast-enhanced ultrasound Liver Imaging Reporting and Data System category M: a systematic review and meta-analysis}

\author{
Jaeseung Shin, Sunyoung Lee, Yeun-Yoon Kim, Yong Eun Chung, Jin-Young Choi, \\ Mi-Suk Park
}

Department of Radiology and Research Institute of Radiological Science, Severance Hospital, Yonsei University College of Medicine, Seoul, Korea

Purpose: A meta-analysis was conducted to determine the proportion of contrast-enhanced ultrasound (CEUS) Liver Imaging Reporting and Data System category M (LR-M) in hepatocellular carcinomas (HCCs) and non-HCC malignancies and to investigate the frequency of individual CEUS LR-M imaging features.

Methods: The MEDLINE and Embase databases were searched from January 1, 2016 to July 23, 2020 for studies reporting the proportion of CEUS LR-M in HCC and non-HCC malignancies. The meta-analytic pooled proportions of HCC and non-HCC malignancies in the CEUS LR-M category were calculated. The meta-analytic frequencies of CEUS LR-M imaging features in nonHCC malignancies were also determined. Risk of bias and applicability were evaluated using the Quality Assessment of Diagnostic Accuracy Studies-2 tool.

Results: Twelve studies reporting the diagnostic performance of the CEUS LR-M category were identified, as well as seven studies reporting the frequencies of individual CEUS LR-M imaging features. The pooled proportions of HCC and non-HCC malignancies in the CEUS LR-M category were $54 \%$ (95\% confidence interval $[\mathrm{Cl}], 44 \%$ to $65 \%)$ and $40 \%(95 \% \mathrm{Cl}, 28 \%$ to $53 \%$ ), respectively. The pooled frequencies of individual CEUS LR-M imaging features in non-HCC malignancies were $30 \%(95 \% \mathrm{Cl}, 17 \%$ to $45 \%)$ for rim arterial phase hyperenhancement, $79 \%$ (95\% Cl, 66\% to $90 \%)$ for early ( $<60 \mathrm{~s})$ washout, and $42 \%(95 \% \mathrm{Cl}, 21 \%$ to $64 \%)$ for marked washout.

Conclusion: In total, $94 \%$ of CEUS LR-M lesions were malignancies, with HCCs representing $54 \%$ and non-HCC malignancies representing $40 \%$. The frequencies of individual CEUS LR-M imaging features varied; early washout showed the highest frequency for non-HCC malignancies.

Keywords: Diagnosis; Liver neoplasms; Microbubbles; Sensitivity and specificity; Ultrasound Key points: Pooled proportions of hepatocellular carcinomas (HCCs) and non-HCC malignancies in contrast-enhanced ultrasound (CEUS) Liver Imaging Reporting and Data System category M (LR-M) were $54 \%$ and $40 \%$. Frequencies of individual LR-M imaging features for CEUS were variable, with early washout showing the highest frequency in non-HCC malignancies.

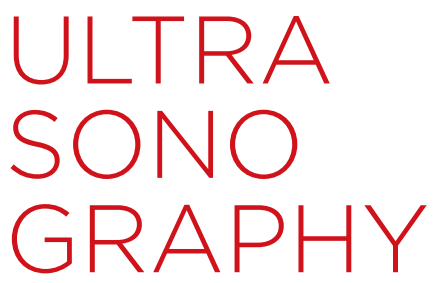

META-ANALYSIS

https://doi.org/10.14366/usg.21011 pISSN: 2288-5919 • elSSN: 2288-5943 Ultrasonography 2022;41:74-82

Received: January 16, 2021

Revised: May 2, 2021

Accepted: May 19, 2021

Correspondence to:

Sunyoung Lee, MD, PhD, Department of Radiology and Research Institute of Radiological Science, Severance Hospital, Yonsei University College of Medicine, 50-1 Yonsei-ro, Seodaemungu, Seoul 03722, Korea

Tel. $+82-2-2228-7400$

Fax. +82-2-2227-8337

E-mail: carnival0126@gmail.com

This is an Open Access article distributed under the terms of the Creative Commons Attribution NonCommercial License (http://creativecommons.org/ licenses/by-nc/4.0/) which permits unrestricted noncommercial use, distribution, and reproduction in any medium, provided the original work is properly cited.

Copyright (C) 2022 Korean Society of Ultrasound in Medicine (KSUM)

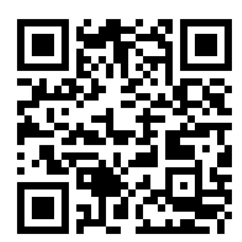

How to cite this article:

Shin J, Lee S, Kim YY, Chun YE, Choi JY, Park MS. Contrast-enhanced ultrasound Liver Imaging Reporting and Data System category $\mathrm{M}$ : a systematic review and meta-analysis. Ultrasonography. 2022 Jan;41(1):74-82. 


\section{Introduction}

Contrast-enhanced ultrasonography (CEUS) is an advanced form of ultrasonography that provides dynamic, real-time evaluations using intravenously injected microbubble contrast agents [1]. The CEUS Liver Imaging Reporting and Data System (LI-RADS) is a comprehensive system for standardizing the terminology, techniques, interpretation, reporting, and data collection of CEUS examinations for the evaluation of focal hepatic lesions detected in patients at high risk for hepatocellular carcinoma (HCC) [2]. Although the fundamental concepts and principles of CEUS LI-RADS are similar to those of computed tomography (CT)/magnetic resonance imaging (MRI) LI-RADS, the algorithms are not identical, reflecting intrinsic differences between the imaging modalities and their corresponding contrast agents [3].

For both the CT/MRI LI-RADS and the CEUS LI-RADS, LI-RADS category $M(L R-M)$ is defined as an observation that is probably or definitely malignant but not specific for HCC $[1,4-6]$. This unique category was designed to preserve the specificity of imaging-based diagnoses of HCC, while maintaining sensitivity for diagnoses of all malignancies [7]. Purely intravascular microbubble contrast agents on CEUS lead to differences in the dynamics and enhancement patterns compared to CT imaging and MRI; thus, the CEUS LI-RADS evaluates different diagnostic imaging features [2]. For the CT/MRI LI-RADS, the LR-M criteria include a targetoid morphology (i.e., rim arterial phase hyperenhancement [APHE], peripheral washout, delayed central enhancement, targetoid restriction, and targetoid transitional or hepatobiliary phase appearance) and a non-targetoid mass (i.e., infiltrative appearance, marked diffusion restriction, necrosis, or severe ischemia) that does not meet the LR-5 criteria $[5,6]$. For the CEUS LI-RADS, LR-M criteria include rim APHE and a targetoid morphology, which is also evaluated in the CT/MRI LIRADS. However, unlike the CT/MRI LI-RADS, early (within $60 \mathrm{~s}$ ) washout and marked washout (black or punched out) are LR-M imaging features evaluated in the CEUS LI-RADS [1].

A recent meta-analysis reported the pooled percentages of $\mathrm{HCC}$ and non-HCC malignancies in the LR-M category using MRI [8]. However, no previous meta-analysis has evaluated the pooled proportions of HCC and non-HCC malignancies categorized as CEUS LR-M or the frequency of each CEUS LR-M imaging feature. Therefore, the purpose of this study was to determine the proportion of the CEUS LR-M category in HCC and non-HCC malignancies and to determine the frequency of each CEUS LR-M imaging feature using CEUS LI-RADS versions 2016 and 2017.

\section{Materials and Methods}

This meta-analysis was conducted in accordance with the Preferred Reporting Items for Systematic Reviews and Meta-Analyses of Diagnostic Test Accuracy Studies statement [9]. The protocol was registered in the International Prospective Register of Systematic Reviews (PROSPERO) (CRD42020218653).

\section{Literature Search Strategy}

We performed a comprehensive search of the MEDLINE and Embase databases. The search date range was January 1, 2016 to July 23, 2020 (i.e., from the publication of the CEUS LI-RADS version 2016 to the most recent search date). The detailed search strategy is presented in Supplementary Table 1. Abstract archives from annual meetings of the Radiological Society of North America, European Society of Radiology, and European Society of Gastrointestinal and Abdominal Radiology were also searched. We excluded case reports, reviews, meta-analyses, letters, commentaries, and errata.

\section{Study Selection}

After duplicates were removed, two independent reviewers screened article titles and abstracts. The full texts of articles with titles or abstracts considered potentially relevant were retrieved and reviewed in duplicate. Discrepancies were resolved by consensus and confirmed by a third reviewer.

\section{Data Extraction}

The following variables were extracted from individual studies: (1) study characteristics (first author, year and nation of publication, study design, and subject enrollment); (2) study population characteristics (number of patients, age, and sex); (3) CEUS LI-RADS version; (4) reference standard; and (5) outcomes of interest. The following outcomes were extracted for each study: the number of observations categorized as CEUS LR-M; the number of HCCS, nonHCC malignancies, and benign lesions categorized as CEUS LR$\mathrm{M}$; and the frequency of each LR-M imaging feature (rim APHE, early washout, and marked washout) for non-HCC malignancies. For studies reporting results from more than one image reviewer, consensus data or averaged data were used. Two authors independently performed data extraction, and disagreements were reevaluated at a consensus meeting in the presence of a third reviewer.

\section{Methodological Quality and Risk of Bias Assessment}

The risk of bias and concerns regarding applicability were evaluated for each study using the Quality Assessment of Diagnostic Accuracy Studies (QUADAS)-2 [10]. The following domains were assessed: 
patient and observation selection, the index test, the reference standard, and the flow and timing.

\section{Statistical Analysis}

The pooled proportions of HCC and non-HCC malignancies categorized as CEUS LR-M and their 95\% confidence intervals (Cls) were calculated using a random-effects model with the inverse variance method and restricted maximum-likelihood estimation. Heterogeneity was assessed using the Cochrane $Q$ test and $\mathrm{I}^{2}$. Meta-regression analysis was performed to explore potential sources of heterogeneity. The pooled frequencies of individual CEUS LR-M imaging features for non-HCC malignancies were also assessed using a random-effects model. Funnel plots and rank tests were used to evaluate publication bias. Statistical analyses were performed using the 'meta' package of $R$ version 3.4.3 ( $R$ Foundation for Statistical Computing, Vienna, Austria).

\section{Results}

The initial search resulted in 248 studies, of which 25 articles and two annual meeting abstracts were reviewed. Fifteen articles were excluded after full-text review (Supplementary Table 2). The remaining 10 eligible articles [11-20] and two annual meeting abstracts $[21,22]$ reported the probabilities of HCCs and non-HCC malignancies in the CEUS LR-M category (Fig. 1). Among these, seven articles $[12,14-17,19,20]$ also reported the frequencies of individual CEUS LR-M imaging features (i.e., rim APHE, early washout, and marked washout).

The characteristics of the included studies are summarized in Table 1. Of the 12 included studies, three studies $[11,20,21]$ had prospective study designs and nine studies [12-19,22] had retrospective study designs. There was only one case-control study [14], while the others were cohort studies [11-13,15-22]. For subject enrollment, eight studies $[11,12,15,18-22]$ consecutively enrolled subjects, while the other four studies $[13,14,16,17]$ selectively included subjects. Eight studies $[13-18,20,22]$ reported hepatitis $B$ virus infection as the predominant etiology, while four studies $[11,12,19,21]$ reported other predominant etiologies such as hepatitis $C$ virus infection or alcohol abuse. Four articles used CEUS LI-RADS version $2016[11-13,19]$ and the other eight studies [14-18,20-22] used CEUS LI-RADS version 2017. Regarding the reference standard, five studies $[13-15,20,22]$ used only the pathological diagnosis, while seven studies $[11,12,16-19,21]$ used a combination of pathological diagnosis and composite imaging or clinical follow-up.

The methodological quality of the studies assessed by the QUADAS-2 is presented in Supplementary Fig. 1. Of the four domains, the flow and timing domain had notable quality concerns, with $75 \%$ of studies having high $[11,20,22]$ or unclear [13$16,19,21]$ risk of bias because they did not use the same reference standard or because the interval between the index test and reference standard was unclear or not stated. In the patient and observation selection domain, $17 \%[13,14]$ and $33 \%[13,14,16,17]$ of the studies had a high risk of bias and high concerns regarding

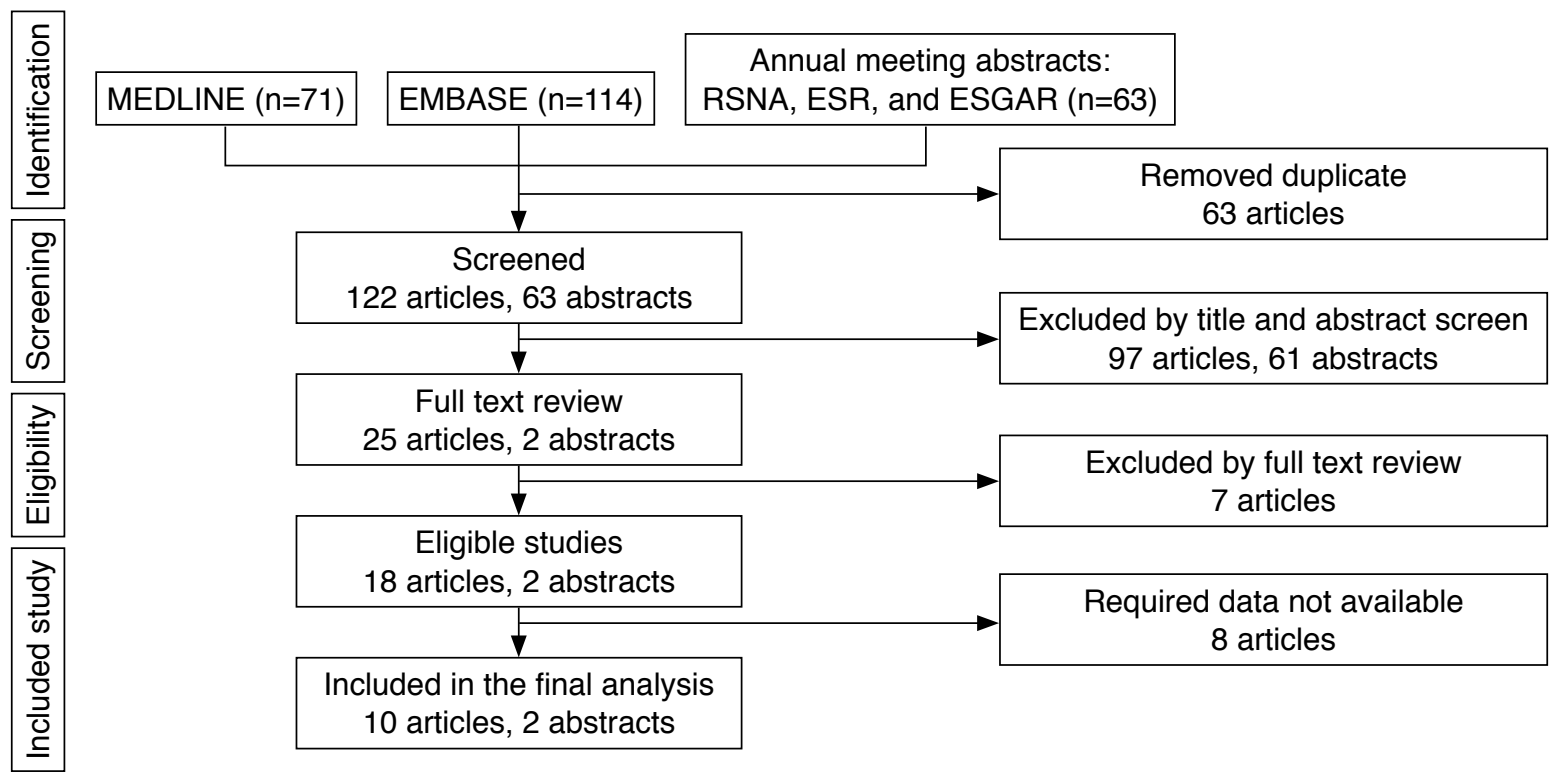

Fig. 1. Flow diagram of study selection. ESGAR, European Society of Gastrointestinal and Abdominal Radiology; ESR, European Society of Radiology; RSNA, Radiological Society of North America. 
Table 1. Characteristics of the included studies

\begin{tabular}{|c|c|c|c|c|c|c|c|c|c|c|c|}
\hline Study & Year & Nation & Study design & $\begin{array}{l}\text { Study } \\
\text { type }\end{array}$ & $\begin{array}{c}\text { Subject } \\
\text { enrollment }\end{array}$ & $\begin{array}{c}\text { No. of } \\
\text { patients }\end{array}$ & $\begin{array}{c}\text { Age } \\
\text { (year) }\end{array}$ & $\begin{array}{c}\text { Men } \\
(\%)\end{array}$ & $\begin{array}{c}\text { Dominant } \\
\text { etiology }\end{array}$ & $\begin{array}{c}\text { CEUS LI-RADS } \\
\text { version }\end{array}$ & Reference standard \\
\hline $\begin{array}{l}\text { Schellhaas et } \\
\text { al. [11] }\end{array}$ & 2017 & Germany & Prospective & Cohort & Consecutive & 100 & $\begin{array}{c}66 \\
(42-85)\end{array}$ & 85 & Non-HBV & 2016 & $\begin{array}{l}\text { Pathology and } \\
\text { composite imaging } \\
\text { or clinical follow-up }\end{array}$ \\
\hline Terzi et al. [12] & 2018 & Italy & Retrospective & Cohort & Consecutive & 848 & $\begin{array}{c}70 \\
(31-89)\end{array}$ & 54 & Non-HBV & 2016 & $\begin{array}{l}\text { Pathology and } \\
\text { composite imaging } \\
\text { or clinical follow-up }\end{array}$ \\
\hline Ling et al. [13] & 2018 & China & Retrospective & Cohort & Selective & 56 & 53 & 82 & HBV & 2016 & Pathology \\
\hline Chen et al. [14] & 2019 & China & Retrospective & $\begin{array}{l}\text { Case- } \\
\text { control }\end{array}$ & Selective & 210 & $\begin{array}{c}55 \\
(32-84)\end{array}$ & 82 & HBV & 2017 & Pathology \\
\hline Li et al. [15] & 2019 & China & Retrospective & Cohort & Consecutive & 1,366 & $52 \pm 12$ & 80 & HBV & 2017 & Pathology \\
\hline $\begin{array}{l}\text { Lyshchik et al. } \\
\text { [21] }\end{array}$ & 2019 & USA & Prospective & Cohort & Consecutive & NR & NR & NR & Non-HBV & 2017 & $\begin{array}{l}\text { Pathology and } \\
\text { composite imaging } \\
\text { or clinical follow-up }\end{array}$ \\
\hline $\begin{array}{l}\text { Zheng et al. } \\
{[16]}\end{array}$ & 2020 & China & Retrospective & Cohort & Selective & 1,826 & $54 \pm 12$ & 90 & HBV & 2017 & $\begin{array}{l}\text { Pathology and } \\
\text { composite imaging } \\
\text { or clinical follow-up }\end{array}$ \\
\hline $\begin{array}{l}\text { Huang et al. } \\
\text { [17] }\end{array}$ & 2020 & China & Retrospective & Cohort & Selective & 172 & $\begin{array}{c}52 \pm 11 \\
(21-82)^{a)}\end{array}$ & 79 & HBV & 2017 & $\begin{array}{l}\text { Pathology and } \\
\text { composite imaging } \\
\text { or clinical follow-up }\end{array}$ \\
\hline Tan et al. [18] & 2020 & Singapore & Retrospective & Cohort & Consecutive & 45 & $\begin{array}{c}63 \\
(34-84)\end{array}$ & 71 & HBV & 2017 & $\begin{array}{l}\text { Pathology and } \\
\text { composite imaging } \\
\text { or clinical follow-up }\end{array}$ \\
\hline $\begin{array}{l}\text { Makoyeva et al. } \\
\text { [19] }\end{array}$ & 2020 & Canada & Retrospective & Cohort & Consecutive & 184 & $\begin{array}{c}62 \\
(27-87)\end{array}$ & 75 & Non-HBV & 2016 & $\begin{array}{l}\text { Pathology and } \\
\text { composite imaging } \\
\text { or clinical follow-up }\end{array}$ \\
\hline Zhou et al. [20] & 2020 & China & Prospective & Cohort & Consecutive & 96 & $\begin{array}{c}59 \\
(35-87)\end{array}$ & 84 & HBV & 2017 & Pathology \\
\hline Ding et al. [22] & 2020 & China & Retrospective & Cohort & Consecutive & 270 & $\begin{array}{c}52 \pm 11 \\
(21-82)^{a}\end{array}$ & NR & HBV & 2017 & Pathology \\
\hline
\end{tabular}

CEUS, contrast-enhanced ultrasonography; LI-RADS, Liver Imaging Reporting and Data System; HBV, hepatitis B virus; NR, not reported.

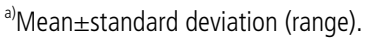

applicability, respectively.

\section{Proportion of HCC and Non-HCC Malignancies in CEUS LR-M}

The proportions of HCC and non-HCC malignancies in the CEUS LR-M category are summarized in Fig. 2. Among 5,728 observations, there were a total of 1,046 LR-M observations in 12 studies, of which 579 were HCCS, 432 were non-HCC malignancies, and 35 were benign. The pooled proportions of HCC and non-HCC malignancies in the CEUS LR-M category were $54 \%(95 \% \mathrm{Cl}$, $44 \%$ to $\left.65 \% ; I^{2}=84 \% ; P<0.001\right)$ and $40 \%(95 \% \mathrm{Cl}, 28 \%$ to $\left.53 \% ; I^{2}=88 \% ; P<0.001\right)$, respectively. After excluding one casecontrol study [14], the pooled proportions of HCC and non-HCC malignancies in the CEUS LR-M category were $59 \%(95 \% \mathrm{Cl}, 52 \%$ to $\left.65 \% ; I^{2}=44 \% ; P=0.056\right)$ and $36 \%(95 \% \mathrm{Cl}, 26 \%$ to $46 \%$, $\mathrm{I}^{2}=66 \% ; \mathrm{P}<0.001$ ), respectively (Supplementary Fig. 2).

Of the 298 non-HCC malignancies with specific diagnoses identified in nine studies [11-14,17,19,21], there were 201 intrahepatic cholangiocarcinomas, 62 metastases, 28 combined hepatocellular cholangiocarcinomas, and three lymphomas (Table 2). No specific diagnoses were available for 134 non-HCC malignancies identified in three studies $[15,20,22]$. For the 35 benign lesions categorized as CEUS LR-M, eight were specified in four studies $[13,16,17,19]$, including two bile duct adenomas, two reactive lymphoid hyperplasias, one inflammatory pseudotumor, one liver abscess, one hemangioma, and one regenerative nodule (Table 2).

\section{Meta-regression Analysis}

For the 10 covariates included in the meta-regression analysis (Table 3), the nation of publication and dominant etiology were significant factors contributing to heterogeneity. The proportion of non-HCC malignancies in the CEUS LR-M category was significantly lower in studies from Asian countries than in studies from Western countries (35\%; $95 \% \mathrm{Cl}, 22 \%$ to $51 \%$ vs. $54 \%$; $95 \% \mathrm{Cl}, 46 \%$ to 
Table 2. Summarized diagnoses for CEUS LI-RADS category M observations

\begin{tabular}{|c|c|c|c|c|c|c|}
\hline Study & $\begin{array}{c}\text { Total No. of LR-M } \\
\text { observations }\end{array}$ & $\begin{array}{c}\text { No. of } \\
\text { HCCs (\%) }\end{array}$ & $\begin{array}{l}\text { No. of non-HCC } \\
\text { malignancies (\%) }\end{array}$ & $\begin{array}{l}\text { Non-HCC malignancy } \\
\text { diagnoses }(n)\end{array}$ & $\begin{array}{l}\text { No. of benign } \\
\text { lesions (\%) }\end{array}$ & Benign diagnoses $(n)$ \\
\hline Schellhaas et al. [11] & 6 & $3(50)$ & $3(50)$ & iCCA (3) & 0 & - \\
\hline Terzi et al. [12] & 82 & $39(48)$ & $43(52)$ & $\begin{array}{l}\text { iCCA (31), cHCC-CCA (8), } \\
\text { metastasis (2), lymphoma (2) }\end{array}$ & 0 & - \\
\hline Ling et al. [13] & 9 & $7(78)$ & $1(11)$ & iCCA (1) & $1(11)$ & Reactive lymphoid hyperplasia (1) \\
\hline Chen et al. [14] & 138 & $38(28)$ & $100(72)$ & iCCA (100) & 0 & - \\
\hline Lyshchik et al. [21] & 11 & $5(45)$ & $6(55)$ & iCCA (2), metastasis (4) & 0 & - \\
\hline Zheng et al. [16] & 354 & $224(63)$ & $127(36)$ & $\begin{array}{l}\text { iCCA (54), cHCC-CCA (20), } \\
\text { metastasis (50), others (3) }\end{array}$ & $3(1)$ & $\begin{array}{l}\text { Biliary adenoma (1), inflammatory } \\
\text { pseudotumor (1), liver abscess (1) }\end{array}$ \\
\hline Huang et al. [17] & 20 & $15(75)$ & $2(10)$ & iCCA (2) & $3(15)$ & $\begin{array}{l}\text { Biliary adenoma (1), hemangioma (1), } \\
\text { reactive lymphoid hyperplasia (1) }\end{array}$ \\
\hline Zhou et al. [20] & 43 & $25(58)$ & $17(40)$ & Not specified & $1(2)$ & Not specified \\
\hline Ding et al. [22] & 68 & $41(60)$ & $22(32)$ & Not specified & $5(8)$ & Not specified \\
\hline
\end{tabular}

CEUS, contrast-enhanced ultrasonography; LI-RADS, Liver Imaging Reporting and Data System; LR-M, Liver Imaging Reporting and Data System category M; HCC, hepatocellular carcinoma; iCCA, intrahepatic cholangiocarcionoma; CHCC-CCA, combined hepatocellular cholangiocarcinoma.

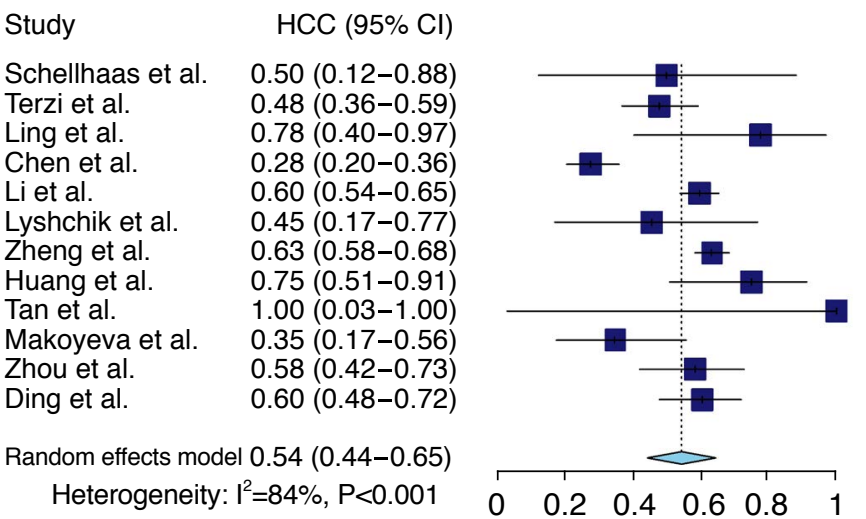

A

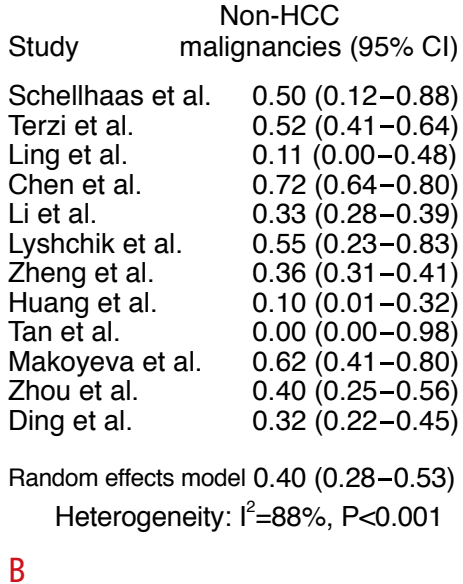

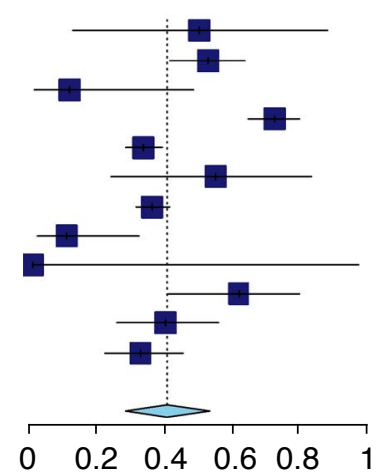

$\begin{array}{llllll}0 & 0.2 & 0.4 & 0.6 & 0.8 & 1\end{array}$

Fig. 2. Forest plots of the pooled proportions of $H C C(A)$ and non-HCC malignancies (B) in the CEUS LR-M category.

HCC, hepatocellular carcinoma; Cl, confidence interval; CEUS, contrast-enhanced ultrasonography; LR-M, Liver Imaging Reporting and Data System category M.

$63 \% ; P=0.035)$. Studies in which hepatitis B viral infection was the dominant etiologies showed lower proportions of non-HCC malignancies in the CEUS LR-M categories than studies with other dominant etiologies (35\%; $95 \% \mathrm{Cl}, 22 \%$ to $51 \%$ vs. $54 \%$; $95 \% \mathrm{Cl}$, $46 \%$ to $63 \% ; P=0.035)$.

\section{Frequencies of CEUS LR-M Imaging Features in Non-HCC Malignancies}

The frequencies of individual imaging features of the CEUS LR-M criteria are summarized in Fig. 3 and Supplementary Table 3. Of the three imaging features, early washout showed the highest metaanalytic pooled frequency in both non-HCC malignancies (79\%; $95 \% \mathrm{Cl}, 66 \%$ to $90 \%)$ and $\mathrm{HCCs}(15 \% ; 95 \% \mathrm{Cl}, 7 \%$ to $26 \%)$. Rim APHE and marked washout showed pooled frequencies of $30 \%$ ( $95 \% \mathrm{Cl}, 17 \%$ to $45 \%)$ and $42 \%(95 \% \mathrm{Cl}, 21 \%$ to $64 \%)$ in non-HCC malignancies, respectively, and $1 \%(95 \% \mathrm{Cl}, 0 \%$ to $1 \%)$ and $3 \%(95 \%$ $\mathrm{Cl}, 2 \%$ to $6 \%$ ) in HCC, respectively (Supplementary Table 3). 
Table 3. Results of the meta-regression analysis of CEUS LI-RADS category M for the proportion of HCC and non-HCC malignancies

\begin{tabular}{|c|c|c|c|}
\hline & \multicolumn{3}{|c|}{ Meta-analytic summary estimates } \\
\hline & $\begin{array}{c}\mathrm{HCC} \\
(95 \% \mathrm{Cl}, \%)\end{array}$ & $\begin{array}{c}\text { Non-HCC malignancy } \\
(95 \% \mathrm{Cl}, \%)\end{array}$ & P-value \\
\hline Year of publication & & & 0.274 \\
\hline Before 2020 & $49(34-64)$ & $47(29-65)$ & \\
\hline After 2020 & $61(50-71)$ & $32(18-49)$ & \\
\hline Nation of publication & & & 0.035 \\
\hline Asian & $58(46-70)$ & $35(22-51)$ & \\
\hline Western & $45(36-54)$ & $54(46-63)$ & \\
\hline Study design & & & 0.735 \\
\hline Prospective & $55(42-67)$ & $43(31-56)$ & \\
\hline Retrospective & $54(42-66)$ & $40(26-55)$ & \\
\hline Subject enrollment & & & 0.562 \\
\hline Consecutive & $54(47-61)$ & $43(34-52)$ & \\
\hline Selective & $59(34-80)$ & $31(9-67)$ & \\
\hline Age (year) & & & 0.096 \\
\hline$\geq 60$ & $44(33-56)$ & $55(43-66)$ & \\
\hline$<60$ & $58(43-72)$ & $35(18-54)$ & \\
\hline Men (\%) & & & 0.850 \\
\hline$\geq 80$ & $55(40-69)$ & $41(25-59)$ & \\
\hline$<80$ & $55(30-79)$ & $35(6-71)$ & \\
\hline Dominant etiology & & & 0.035 \\
\hline HBV & $58(46-70)$ & $35(22-51)$ & \\
\hline Non-HBV & $45(36-54)$ & $54(46-63)$ & \\
\hline CEUS LI-RADS version & & & 0.486 \\
\hline Version 2016 & $53(37-69)$ & $42(21-67)$ & \\
\hline Version 2017 & $54(42-65)$ & $42(29-56)$ & \\
\hline Reference standard & & & 0.932 \\
\hline Pathology only & $54(38-69)$ & $40(23-60)$ & \\
\hline Pathology or CCRS & $54(42-66)$ & $43(30-58)$ & \\
\hline $\begin{array}{l}\text { Patient and observation } \\
\text { selection in QUADAS-2 }\end{array}$ & & & 0.732 \\
\hline Low risk & $58(48-68)$ & $42(32-52)$ & \\
\hline High or unclear risk & $61(36-85)$ & $38(15-64)$ & \\
\hline
\end{tabular}

CEUS, contrast-enhanced ultrasonography; LI-RADS, Liver Imaging Reporting and Data System; HCC, hepatocellular carcinoma; $\mathrm{Cl}$, confidence interval; HBV, hepatitis $B$ virus; CCRS, composite clinical reference standard; QUADAS-2, Quality Assessment of Diagnostic Accuracy Studies-2.

\section{Publication Bias}

No significant publication bias was identified across the studies $(\mathrm{P}=0.784$ ) (Supplementary Fig. 3).

\section{Discussion}

In this meta-analysis, the pooled proportion of overall malignancies in the CEUS LR-M category was $94 \%$, with HCCs representing $54 \%$ and non-HCC malignancies representing $40 \%$. Substantial heterogeneity was identified, with the nation of publication and dominant etiology being significant factors contributing to this heterogeneity. The frequencies of individual CEUS LR-M imaging features varied, with early washout showing the highest frequency for non-HCC malignancies.

The present meta-analysis revealed that the proportion of overall malignancies in the CEUS LR-M category was high (94\%), with similarity to the proportion of the CT/MRI LR-M (93\%) category reported in a previous meta-analysis [23]. However, the pooled proportion of HCC in the LR-M category was higher using the CEUS LI-RADS (54\%) than the CT/MRI LI-RADS $(22 \%-36 \%)$, and the pooled proportion of non-HCC malignancies was lower using the CEUS LI-RADS (40\%) than the CT/MRI LI-RADS (57\%-77\%) $[8,23,24]$. This discrepancy in the pooled proportion between CEUS and CT/MRI LI-RADS was similar in the subgroup analysis of the present study that included only cohort studies (HCC 59\% and non-HCC malignancies 36\% in the CEUS LR-M). While CEUS LR-M shares some fundamental concepts and definitions with CT/MRI LR-M, there are also some dissimilarities between these categories, reflecting intrinsic differences in image acquisition (i.e., real-time imaging vs. non-real-time imaging) and contrast material properties (i.e., purely intravascular microbubbles vs. low-molecularweight agents) [2]. Accordingly, the imaging features comprising the LR-M criteria differ substantially between CEUS and CT/MRI LIRADS, except for rim APHE. The CT/MRI LR-M criteria encompass a targetoid morphology (i.e., rim APHE, peripheral "washout," delayed central enhancement, targetoid restriction, and targetoid transitional or hepatobiliary phase appearance) and a non-targetoid mass (i.e., infiltrative appearance, marked diffusion restriction, necrosis, or severe ischemia) that does not meet the LR-5 criteria $[5,6]$. By contrast, the CEUS LR-M criteria have unique imaging features (i.e., early washout and marked washout) in addition to rim APHE $[1,4]$. These differences may have led to discrepancies in the proportions of HCC and non-HCC malignancies categorized as LR-M using CEUS versus CT/MRI. Although the proportions of HCC in the LR-M category are different between CEUS and CT/MRI, classifying atypical HCCs as LR-M may not generally be clinically problematic, because LI-RADS recommends that such lesions should be biopsied to obtain an accurate pathologic diagnosis.

In this meta-analysis, substantial heterogeneity was identified among the included studies, with meta-regression analysis revealing that the nation of publication and dominant etiology significantly contributed to this heterogeneity. Studies from Asian countries had a higher proportion of HCCS and a lower proportion of non-HCC malignancies in the CEUS LR-M category than those from Western 


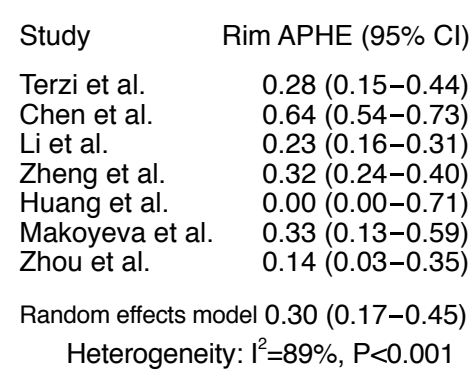

A

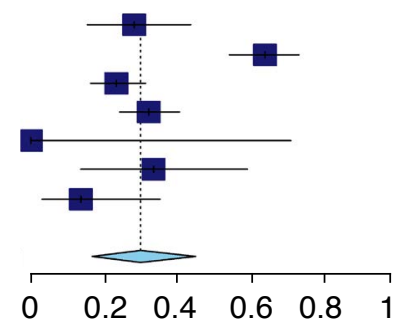

$\begin{array}{lr}\text { Study } & \begin{array}{c}\text { Marked } \\ \text { washout }(95 \% \mathrm{Cl})\end{array} \\ \text { Terzi et al. } & 0.81(0.67-0.92) \\ \text { Chen et al. } & 0.59(0.49-0.69) \\ \text { Li et al. } & 0.57(0.48-0.65) \\ \text { Zheng et al. } & 0.13(0.08-0.20) \\ \text { Huang et al. } & 0.33(0.01-0.91) \\ \text { Makoyeva et al. } & 0.28(0.10-1.53) \\ \text { Zhou et al. } & 0.18(0.05-0.40) \\ \text { Random effects model } 0.42(0.21-0.64)\end{array}$

C

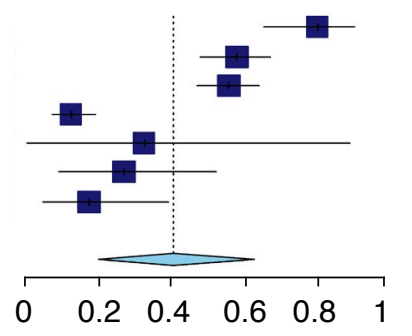

countries. This finding may be associated with geographic differences in the dominant etiologies of underlying liver disease, with hepatitis $B$ being more common in Asian countries and hepatitis $C$ or alcohol abuse predominating in Western countries $[25,26]$. Chronic hepatitis $B$ viral infection, either with or without cirrhosis, is the dominant etiology in Asian countries, while cirrhosis because of other causes (e.g., chronic hepatitis $C$ viral infection or alcohol abuse) are more commonly noted in non-Asian countries [27]. Although the underlying mechanism is not understood, the presence or degree of cirrhosis may influence the interpretation of the imaging features of hepatic focal lesions on CEUS. Further studies are needed to understand the proportions of HCC and non-HCC malignancies in the CEUS LR-M category based on the underlying liver disease.

This study demonstrated that the pooled frequencies of CEUS LR-M imaging features in non-HCC malignancies varied. Of the three CEUS LR-M criteria, early washout had the highest pooled frequency (79\%). In a previous study [19], early washout was the only predictive CEUS LR-M feature for non-HCC malignancies in multivariable logistic regression analysis. Meanwhile, rim APHE on CEUS showed the lowest pooled frequency (30\%) in non-HCC malignancies. This finding contrasts with the results of a previous meta-analysis using CT/MRI, where rim APHE had the highest frequency in non-HCC malignancies [8]. Differences in imaging modalities and contrast agent properties may have contributed to the observed differences in the frequencies of imaging features using CEUS or CT/MRI. While the interpretation using CT/MRI relies

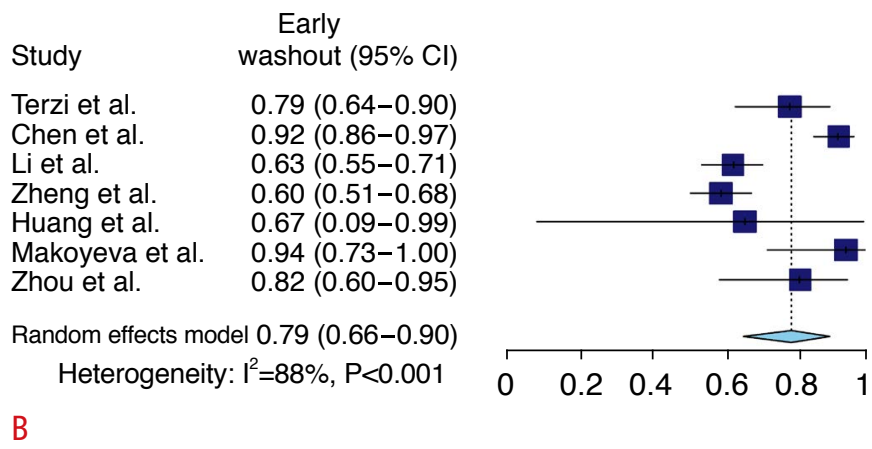

Fig. 3. Forest plots of the pooled frequencies of CEUS LR-M imaging features for non-HCC malignancies.

These imaging features included rim arterial phase hyperenhancement (A), early washout (B), and marked washout (C). APHE, arterial phase hyperenhancement; $\mathrm{Cl}$, confidence interval; CEUS, contrast-enhanced ultrasonography; LR-M, Liver Imaging Reporting and Data System category M; HCC, hepatocellular carcinoma. on static phases, CEUS permits a real-time assessment [2]. This provides superior temporal resolution compared to CT/MRI and also virtually prevents imprecise timing of the arterial phase $[1,2]$. In addition, the appearance of washout on CEUS reflects true washout, which can be measured using a quantitative time-intensity curve $[28,29]$, whereas that on CT/MRI may reflect increased enhancement of surrounding parenchyma rather than a true decline of tumor enhancement [1]. The purely intravascular contrast agents used for CEUS cannot pass through the vascular endothelial fenestration; thus, non-HCC malignancies, such as intrahepatic cholangiocarcinoma, show early onset and marked degree of washout due to low blood volume in the tumor $[1,30]$. In contrast, the low-molecular-weight contrast agents used for CT/MRI can pass through the permeable endothelium into the interstitial space and gradually accumulate in the centrally located fibrous stroma, which results in delayed central enhancement and peripheral washout in non-HCC malignancies $[1,30]$.

This study has several limitations. First, substantial heterogeneity was noted, potentially affecting the general applicability of our summary estimates. To identify potential factors contributing to this heterogeneity, a meta-regression analysis was performed; however, this issue remained a matter of concern. Second, a limited number of prospective studies were available for our meta-analysis, indicating a risk of selection bias and a major methodological limitation. Third, we could not conduct subgroup analyses based on the size of hepatic observations, the presence of cirrhosis, or the etiologies 
of underlying liver disease because of incomplete reporting and data unavailability. Fourth, this meta-analysis only included studies published in English; therefore, potentially eligible studies published in other languages may have been omitted.

In conclusion, among observations categorized as CEUS-LR-M, 94\% were malignancies, with HCCs representing $54 \%$ and nonHCC malignancies representing $40 \%$. The frequencies of different CEUS LR-M imaging features varied, with early washout showing the highest frequency for non-HCC malignancies.

ORCID: Jaeseung Shin: https://orcid.org/0000-0002-6755-4732; Sunyoung Lee: https:// orcid.org/0000-0002-6893-3136; Yeun-Yoon Kim: https://orcid.org/0000-0003-20185332; Yong Eun Chung: https://orcid.org/0000-0003-0811-9578; Jin-Young Choi: https://orcid.org/0000-0002-9025-6274; Mi-Suk Park: https://orcid.org/0000-00015817-2444

\section{Author Contributions}

Conceptualization: Shin J, Lee S. Data acquisition: Lee S, Kim YY. Data analysis or interpretation: Shin J, Lee S, Chung YE, Choi JY, Park MS. Drafting of the manuscript: Shin J, Lee S. Critical revision of the manuscript: Shin J, Lee S, Kim YY, Chung YE, Choi JY, Park MS. Approval of the final version of the manuscript: all authors.

\section{Conflict of Interest}

No potential conflict of interest relevant to this article was reported.

\section{Supplementary Material}

Supplementary Table 1. Detailed search strategy for PICO (https:// doi.org/10.14366/usg.21011).

Supplementary Table 2. Excluded studies by full text review (https:// doi.org/10.14366/usg.21011).

Supplementary Table 3. Meta-analytic summary frequency of CEUS LR-M imaging features for HCC and non-HCC malignancy (https:// doi.org/10.14366/usg.21011).

Supplementary Fig. 1. Methodological quality of the studies with the Quality Assessment of Diagnostic Accuracy Studies (QUADAS)-2. The results of the included studies in terms of the risk of bias (left) and concerns regarding applicability (right) according to each QUADAS-2 domain (https://doi.org/10.14366/usg.21011).

Supplementary Fig. 2. Subgroup analysis of the pooled proportions of HCC and non-HCC malignancy in the CEUS LR-M category using cohort studies. HCC, hepatocellular carcinoma; $\mathrm{Cl}$, confidence interval; CEUS, contrast-enhanced ultrasound; LR-M, Liver Imaging
Reporting and Data System category M (https://doi.org/10.14366/ usg.21011).

Supplementary Fig. 3. Funnel plot for publication bias (https://doi. org/10.14366/usg.21011).

\section{References}

1. American College of Radiology. CEUS LI-RADS v2017 [Internet]. Reston, VA: American College of Radiology, 2017 [cited 2020 Oct 3]. Available from: https://www.acr.org/Clinical-Resources/Reportingand-Data-Systems/LI-RADS/CEUS-LI-RADS-v2017.

2. Kim TK, Noh SY, Wilson SR, Kono Y, Piscaglia F, Jang HJ, et al. Contrast-enhanced ultrasound (CEUS) liver imaging reporting and data system (LI-RADS) 2017: a review of important differences compared to the CT/MRI system. Clin Mol Hepatol 2017;23:280289.

3. Cunha GM, Fowler KJ, Abushamat F, Sirlin CB, Kono Y. Imaging diagnosis of hepatocellular carcinoma: the Liver Imaging Reporting and Data System, why and how? Clin Liver Dis 2020;24:623-636.

4. American College of Radiology. ACR CEUS LI-RADS 2016 [Internet]. Reston, VA: American College of Radiology, 2016 [cited 2020 Oct 3]. Available from: https://www.acr.org/Clinical-Resources/Reportingand-Data-Systems/LI-RADS/CEUS-LI-RADS-v2016.

5. American College of Radiology. CT/MRI LI-RADS v2017 [Internet]. Reston, VA: American College of Radiology, 2017 [cited 2021 Apr 15]. Available from: https://www.acr.org/Clinical-Resources/Reportingand-Data-Systems/LI-RADS/CT-MRI-LI-RADS-V2017.

6. American College of Radiology. CT/MRI LI_RADS v2018 [Internet]. Reston, VA: American College of Radiology, 2018 [cited 2021 Apr 15]. Available from: https://www.acr.org/Clinical-Resources/Reportingand-Data-Systems/LI-RADS/CT-MRI-LI-RADS-v2018.

7. Fowler KJ, Potretzke TA, Hope TA, Costa EA, Wilson SR. LI-RADS $M(L R-M)$ : definite or probable malignancy, not specific for hepatocellular carcinoma. Abdom Radiol (NY) 2018;43:149-157.

8. Kim DH, Choi SH, Park SH, Kim KW, Byun JH, Kim SY, et al. Liver imaging reporting and data system category $\mathrm{M}$ : a systematic review and meta-analysis. Liver Int 2020;40:1477-1487.

9. McInnes MDF, Moher $D$, Thombs BD, McGrath TA, Bossuyt PM; PRISMA-DTA Group, et al. Preferred reporting items for a systematic review and meta-analysis of diagnostic test accuracy studies: the PRISMA-DTA statement. JAMA 2018;319:388-396.

10. Whiting PF, Rutjes AW, Westwood ME, Mallett S, Deeks JJ, Reitsma JB, et al. QUADAS-2: a revised tool for the quality assessment of diagnostic accuracy studies. Ann Intern Med 2011;155:529-536.

11. Schellhaas B, Gortz RS, Pfeifer L, Kielisch C, Neurath MF, Strobel D. Diagnostic accuracy of contrast-enhanced ultrasound for the differential diagnosis of hepatocellular carcinoma: ESCULAP versus 
CEUS-LI-RADS. Eur J Gastroenterol Hepatol 2017;29:1036-1044.

12. Terzi $E$, lavarone $M$, Pompili $M$, Veronese $L$, Cabibbo $G$, Fraquelli $M$, et al. Contrast ultrasound LI-RADS LR-5 identifies hepatocellular carcinoma in cirrhosis in a multicenter restropective study of 1,006 nodules. J Hepatol 2018;68:485-492.

13. Ling $W$, Wang $M, M a X$, Qiu T, Li J, Lu Q, et al. The preliminary application of liver imaging reporting and data system (LI-RADS) with contrast-enhanced ultrasound (CEUS) on small hepatic nodules ( $\leq 2 \mathrm{~cm}$ ). J Cancer 2018;9:2946-2952.

14. Chen LD, Ruan SM, Lin Y, Liang JY, Shen SL, Hu HT, et al. Comparison between M-score and LR-M in the reporting system of contrast-enhanced ultrasound LI-RADS. Eur Radiol 2019;29:42494257.

15. Li J, Ling W, Chen S, Ma L, Yang L, Lu Q, et al. The interreader agreement and validation of contrast-enhanced ultrasound liver imaging reporting and data system. Eur J Radiol 2019;120:108685.

16. Zheng W, Li Q, Zou XB, Wang JW, Han F, Li F, et al. Evaluation of Contrast-enhanced US LI-RADS version 2017: application on 2020 liver nodules in patients with hepatitis B infection. Radiology 2020;294:299-307.

17. Huang JY, Li JW, Lu Q, Luo Y, Lin L, Shi YJ, et al. Diagnostic accuracy of CEUS LI-RADS for the characterization of liver nodules $20 \mathrm{~mm}$ or smaller in patients at risk for hepatocellular carcinoma. Radiology 2020;294:329-339.

18. Tan Z, Teoh WC, Wong KM, Wansaicheong GK, Sandrasegaran K. Analysis of comparative performance of CEUS and CECT/MR LIRADS classification: Can CEUS dichotomize LI-RADS indeterminate lesions on CT or MRI? Clin Imaging 2020;62:63-68.

19. Makoyeva A, Kim TK, Jang HJ, Medellin A, Wilson SR. Use of CEUS LI-RADS for the accurate diagnosis of nodules in patients at risk for hepatocellular carcinoma: a validation study. Radiol Imaging Cancer 2020;2:e190014.

20. Zhou H, Zhang C, Du L, Jiang J, Zhao Q, Sun J, et al. Contrastenhanced ultrasound Liver Imaging Reporting and Data System in diagnosing hepatocellular carcinoma: diagnostic performance and interobserver agreement. Ultraschall Med 2020 Jul 8 [Epub]. https://doi.org/10.1055/a-1168-6321.

21. Lyshchik A, Kono Y, Piscaglia F, Rodgers SK, Wile GE, Kamaya A, et al. Clinical validation of CEUS LI-RADS in prospective multi- center study: preliminary results. In: 2019 Scientific Assembly and Annual Meeting; 2019 Dec 1-6; Chicago, IL, USA. Oak Brook, IL: Radiological Society of North America, 2020.

22. Ding J, Long L, Zhou H, Zhou Y, Wang Y. The predictive value of the liver imaging reporting and data system with contrast-enhanced ultrasound (v2017) in the risk of hepatocellular carcinoma in a high-risk population. In: ECR 2020: European Congress of Radiology; 2020 Jul 15-19. Vienna: European Congress of Radiology, 2020.

23. van der Pol CB, Lim CS, Sirlin CB, McGrath TA, Salameh JP, Bashir $M R$, et al. Accuracy of the Liver Imaging Reporting and Data System in computed tomography and magnetic resonance image analysis of hepatocellular carcinoma or overall malignancy: a systematic review. Gastroenterology 2019;156:976-986.

24. Lee S, Kim SS, Roh YH, Choi JY, Park MS, Kim MJ. Diagnostic performance of CT/MRI Liver Imaging Reporting and Data System v2017 for hepatocellular carcinoma: a systematic review and metaanalysis. Liver Int 2020;40:1488-1497.

25. European Association for the Study of the Liver. EASL Clinical Practice Guidelines: management of hepatocellular carcinoma. J Hepatol 2018;69:182-236.

26. Marrero JA, Kulik LM, Sirlin CB, Zhu AX, Finn RS, Abecassis MM, et al. Diagnosis, staging, and management of hepatocellular carcinoma: 2018 Practice Guidance by the American Association for the Study of Liver Diseases. Hepatology 2018;68:723-750.

27. Wu J. The changing epidemiology of hepatocellular carcinoma in Asia versus United States and Europe. Adv Mod Oncol Res 2017:3:51-58.

28. Pei XQ, Liu LZ, Liu M, Zheng W, Han F, Li AH, et al. Contrastenhanced ultrasonography of hepatocellular carcinoma: correlation between quantitative parameters and histological grading. $\mathrm{Br} J$ Radiol 2012;85:e740-e747.

29. Pei XQ, Liu LZ, Xiong YH, Zou RH, Chen MS, Li AH, et al. Quantitative analysis of contrast-enhanced ultrasonography: differentiating focal nodular hyperplasia from hepatocellular carcinoma. Br J Radiol 2013;86:20120536.

30. Wilson SR, Lyshchik A, Piscaglia F, Cosgrove D, Jang HJ, Sirlin C, et al. CEUS LI-RADS: algorithm, implementation, and key differences from CT/MRI. Abdom Radiol (NY) 2018;43:127-142. 